\title{
Evaluation of a hub-and-spoke model for the delivery of femtosecond laser assisted cataract surgery within the context of a large randomized controlled trial
}

\author{
H.W. Roberts MSc FRCOphth ${ }^{1,2}$, V.B. Wagh MD FRCOphth ${ }^{1}$, I.J.M Mullens BA ${ }^{1}$, S. Borsci $\mathrm{PhD}^{3}$, \\ M.Z. Ni PhD ${ }^{3}$, D.P.S. O'Brart MD FRCS FRCOphth $\mathrm{DO}^{1,2}$ \\ 1. Department of Ophthalmology, Guy's and St. Thomas' NHS Foundation Trust, London, UK \\ 2. King's College London, London, UK \\ 3. Division of Surgery, Department of Surgery and Cancer, Imperial College London, St \\ Mary's Hospital, London, UK
}

Key Words: cataract, phacoemulsification, productivity, femtosecond laser assisted cataract surgery, National Health Service, high volume

Corresponding author:

Harry Roberts MSc FRCOphth

Department of Ophthalmology,

Guy's and St. Thomas' NHS Foundation Trust,

Lambeth Palace Road,

London SE1 7EH.

England

Email: harry.roberts@nhs.net

Telephone +442071884331

Fax $\quad+442071884318$

Financial disclosure: This research has been supported by a non-commercial research grant from Alcon Incorporated. Grant number: IIT \#17440075. The funding organisation had no role in the design or conduct of this research.

Competing Interests: Professor O'Brart has undertaken consultancy work for Sooft Italia SPA and Alcon Inc in the past 12 months. No other conflicting relationship exists for any author.

Contributorship statement: Authors HWR, VW, IJMM and DOB contributed to acquisition of data, analysis and interpretation of data. Authors HR, MN, SB contributed to analysis of data, all authors contributed to drafting the article and revising it critically for important intellectual content.

This work has not previously been presented or published.

Word count (excluding tables, figures, legends): 4,269 


\section{Synopsis}

A femtosecond laser can improve cataract theatre efficiency by adopting a hub-andspoke model with one laser supplying patients to multiple independent operating rooms running in parallel. 
Aims:

To test a hypothesis that cataract operating room (OR) productivity can be improved with a femtosecond-laser (FL) using a hub-and-spoke model and whether any increase in productivity can offset additional costs relating to the FL.

\section{Methods}

400 eyes of 400 patients were enrolled in a randomised-controlled trial comparing FL-assistedcataract-surgery (FLACS) with conventional-phacoemulsification-surgery (CPS). 299/400 operations were performed on designated high volume theatre lists (FLACS=134,CPS=165), whereby a hub-and-spoke FLACS model $(1 \mathrm{xFL}, 2 \mathrm{xORs}=2: 1)$ was compared to independent CPS theatre lists. Details of operative timings and OR utilisation were recorded. Differences in productivity between hub-and-spoke FLACS and CPS sessions were compared using an economic model including testing hypothetical 3:1 and 4:1 models.

Results

The duration of the operation itself was $12.04 \pm 4.89$ minutes(min) for FLACS compared with CPS of $14.54 \pm 6.1 \mathrm{~min}(\mathrm{p}<0.001)$. Total patient time in the OR was reduced from $23.39 \pm 6.89 \mathrm{~min}$ with CPS to $20.34 \pm 5.82 \mathrm{~min}$ with FLACS( $<<0.001$ )(reduction of $3.05 \mathrm{~min}$ per case). There was no difference in OR turnaround time between the models. Average number of patients treated per theatre list was 9 for FLACS and 8 for CPS. OR utilisation was $92.08 \%$ for FLACS and $95.83 \%$ for CPS ( $\mathrm{p}<0.001)$. Using a previously established economic model, the FLACS service cost $£ 144.60$

more than CPS per case. This difference would be $£ 131$ and $£ 125$ for 3:1 and 4:1 models respectively.

Conclusion The FLACS hub-and-spoke model was significantly faster than CPS, with patients spending less time in the OR. This enabled a improvement in productivity, but insufficient to meaningfully offset the additional costs relating to FLACS.

Abstract word count: 250 


\section{$\underline{\text { Introduction }}$}

Over the past decade, Femtosecond lasers (FLs) have been introduced into the field of cataract surgery to try to automate the procedure and more importantly improve both efficacy and safety ${ }^{1}$. Multiple prospective case series have been published which appear to support its potential in cataract surgery and more surgeons are adopting this new technology ${ }^{1-6}$. A recent Cochrane Review of 16 RCTS including 1638 eyes concluded, 'There is currently not enough evidence to determine the benefits and harms of laser-assisted cataract surgery compared with standard ultrasound cataract surgery. The evidence is uncertain because current studies have not been large enough to provide a reliable answer to this question' 7 .

Until good quality evidence is available describing better clinical outcomes it is not possible to currently support the widespread introduction of FLACS within public healthcare organisations such as the National Health Service (NHS). This is especially pertinent as by the very nature of its complex technology, FLACS has significant associated financial costs, including initial purchase costs of the laser itself, servicing, depreciation and the individual patient interfaces (PI). These costs seriously question its financial viability, especially in healthcare systems funded by the state. In addition, current published studies with FLACS report increased total surgical time and therefore reduced patient turnover and productivity ${ }^{8-10}$. This is because the operating surgeon in these studies is typically performing both the FL treatment as well as the subsequent lens extraction within the operating room (OR). This reduction in productivity, is highly important within the public health sector, where high volume surgical models are necessary to meet the both the increasing numbers of patients requiring cataract surgery and economic limitations. It is of note that, the current published literature on the economics of FLACS mainly originates from healthcare systems within the private sector, where supplementary costs of advanced technologies may to a certain extend be passed onto the patient in the form of a co-payment system ${ }^{8}$. However, even in such healthcare models, the literature advocates that FLACS at this time is not cost-effective ${ }^{8}$.

Despite these considerations, FLACS does offer the promise to automate a number of the component parts of cataract surgery so that they do not need to be undertaken by an appropriately trained ophthalmic surgeon within the OR. Surgical steps such as corneal incisions, arcuate keratotomies, capsulotomies and nuclear lens division can be undertaken with FL by a doctor in training or suitably accredited and trained nurse/technician in a clean room. This has the potential to reduced the amount of time each individual patient spends in the OR with the ophthalmic surgeon. As a result, the efficiency of cataract surgery might be improved by increasing the number of surgical cases undertaken in a given time period. This potential efficiency is increased if a "hub and spoke" model is utilized, with a single FL treating and then feeding patients into several operating ORs for completion of surgery. Potentially, if the number of cases per theatre session can be increased sufficiently then the additional costs associated with FL technology might be offset. In a previous publication, we have previously explored this possibility using a hypothetical model based on real world financial data ${ }^{11}$. We reported that in order to break even, there would need to be, for example, a $43 \%$ increase in the number of operations performed per theatre list accompanied by a need to discount the cost of the PIs by at least $52 \%$ by the manufacturers ${ }^{2}$.

As yet, there are no publications looking at the efficacy, safety and additionally the economics of FLACS compared to CPS, within a 'hub-and-spoke' model, as described above, in a real world public health sector setting, where both trainee and fully accredited surgeons undertake surgery, with all the constraints that can be associated with the Public Health Sector, such as limited financial resources, OR space and resistance to change in formalized working practices In order to investigate some of these issues, we undertook an RCT comparing FLACS with CPS, in which we delivered our FLACS service using a hub-and-spoke model. Surgeries were performed by 3 cataract surgeons of differing levels of experience (one fully accredited with twenty years experience, one 
newly accredited and one specialist registrar). Two hundred and ninety-nine of the 400 cases were performed on designated high volume theatre lists, whereby a hub-and-spoke FLACS model (with one femtosecond laser and two ORs) was compared to independent CPS theatre lists. Details of operative timings and OR utilisation within these lists were recorded. Our aims were to provide the best quality evidence to date on whether FLACS can improve productivity in cataract surgery in the public health sector while maintaining safety and efficacy and what models of FLACS delivery might offet its associated addition costs. 


\section{Methods}

\section{Randomised Controlled Trial}

This analysis of relative productivity of FLACS delivered by hub-and-spoke model vs CPS was performed as a secondary outcome of a prospective randomised interventional case-controlled study at a single University Hospital (Guy's \& St Thomas' Hospital NHS Foundation Trust, London, UK) to compare the clinical outcomes of FLACS with CPS (Clinicaltrials.gov registration number

NCT02825693). The study was approved by local Research \& Development and Cambridge South Research Ethics Committee (reference 16/EE/0180). This study adhered to the tenets of the declaration of Helsinki.

Patients were screened, recruited and consented from routine cataract clinics by members of the trial team (HR, VW) as per the trial protocol (Version 2.0, 18/05/2016) (Table 1).

\begin{tabular}{|c|c|}
\hline Inclusion Criteria: & \\
\hline 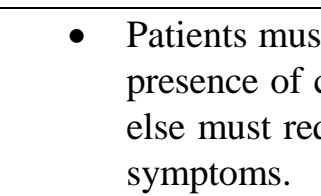 & $\begin{array}{l}\text { have reduced visual acuity or visual symptoms attributed to the } \\
\text { cataract in one or both eyes by the examining ophthalmologist or } \\
\text { quire cataract surgery on clinical grounds other than visual }\end{array}$ \\
\hline $\begin{array}{l}\text { - Patients mus } \\
\text { surgery. }\end{array}$ & t be willing to attend for follow-up at 3-4 weeks after cataract \\
\hline $\begin{array}{l}\text { - Patients mus } \\
\text { completion } \mathrm{c}\end{array}$ & $\begin{array}{l}\text { thave sufficient English language for informed consent and } \\
\text { of the patient reported outcome questionnaires. }\end{array}$ \\
\hline Exclusion Criteria: & \\
\hline - Children bel & ow the age of 18 \\
\hline - Already enrc & lled in another study \\
\hline - Clinical cont & raindications for FLACS, such as: \\
\hline & Significant corneal opacities \\
\hline$\bullet$ & Small pupils $(<4 \mathrm{~mm})$ following pharmacological dilatation \\
\hline & $\begin{array}{l}\text { Patients unable to lie sufficiently flat so as to be positioned } \\
\text { underneath the laser machine. }\end{array}$ \\
\hline
\end{tabular}

Table 1. Inclusion and exclusion criteria for enrolment into the study

\section{Cataract Surgery Delivery Models}

\section{General Model, Staff-duties and Patient Flow}

Cataract operations were performed during 4 hour theatre sessions, either in the morning or afternoon. Patients for cataract surgery were admitted on a staggered arrival basis to an ophthalmic day care unit (ODCU) which staffed by a receptionist and a mixture of ophthalmic technicians (OTs) and ophthalmic nurses (ONs) (Table 4).

After electronic registration by the receptionist, the patients were prepared for the OR by the ON/OTs. This included a series of medical observations, such as blood pressure and blood sugar (if diabetic), and administration of mydriatic therapy. Mydriatic therapy used in this study included a Mydriasert implant (Thea Laboratories, Clermont-Ferrand, France) and two drops of topical diclofenac sodium $0.1 \%$ to reduce the risk of intraoperative miosis ${ }^{12,13}$. The ward ON/OTs brought and collected the patients to and from the OR, which were adjacent to the ODCU, after being telephoned by one of the OR nurses (TNs). After surgery was completed the ON/OTs performed further medical observations, gave advice about aftercare, dispensed medication, discharged the patients and arranged follow-up. 
Patients were treated on either all-FLACS or all-CPS theatre lists. All cataract operations were performed under local anaesthetic. All were unilateral and no other additional procedures planned, other than arcuate keratotomies for reduction of corneal astigmatism.

The duties of the ophthalmologist inside the ORincluded: helping positioning the patient on the operating table, the scribing of patient details onto the whiteboard, the removing of the Mydriasert implant, the marking of the forehead above the eye for caratact surgery prior to the World Health Organization (WHO) checklist $^{14}$, scrubing and gowning, leading the WHO checklist, preparation and draping of the eye for surgery, operating, writing the operation notes, and scanning the paper WHO checklist into the hospital's electronic patient record software.

\section{FLACS Hub and Spoke Delivery Model}

FLACS theatre lists were run as a hub-and-spoke model (figure 1). A LenSx femtosecond laser (Alcon Inc, Fort Worth, Tx, USA) was installed in the anaesthetic room of one of the ORs, hereafter referred to as the laser suite (LS), and was used to feed patients into two adjacent ophthalmic ORs which were running in parallel. The femtosecond laser was operated by an ophthalmologist (HR, VW). The model required an additional OT who supervised patients waiting in the corridor outside the LS(Table 4). There was a maximum of 4 patients seated in the theatre corridor at any one time ( 2 patients waiting for laser treatment, 2 patients waiting to enter OR). In the FLACS model, the ophthalmologist performing the femtosecond laser treatment was responsible for marking the patients' eyes before laser, delivering laser treatment, removing the Mydriasert implant, and instilling additional topical phenylephrine 10\%. Performing laser treatment included preparing the patient interface, entering patient details into the FL, selecting the planned treatment profile, positioning the patient on the laser bed, instilling topical anaesthetic in to the operative eye, inserting the lid speculum, docking the patient interface to the eye, performing Optical Coherence Tomography (OCT) of the anterior segment, choosing the treatment parameters, and delivering the laser treatment. If completion of FL treatment was not possible for any reason, this and the reason why was recorded and the patient proceeded to the OR for CPS.

The number of patients booked to each 4 hour theatre list were decided in advance of each theatre list. The intention was to attempt to always maximize the number of patients treated during the allotted theatre time with reference to the levels of nursing and para-medical staffing. Initial targets were chosen based on existing experience of CPS and FLACS at our institution and titrated as the trial progressed, according to whether theatre lists were finishing early or over-running.

\section{Operating Room Timings}

Two hundred and ninety-nine of the 400 cases were performed on designated high volume theatre lists, whereby patients were randomized to either hub-and-spoke FLACS model (with one FL and two ORs) or CPS only theatre lists. Various timings of OR utilization were undertaken by a TN and included the time taken for the patient to enter the OR, duration of cataract surgery, time taken for the patient to exit the OR after completion of surgery, the total individual patient time in the OR, the time the OR was empty between patients, over and under-runs of allotted OR time, etc

Timings of patient entry to the OR, start of operation, end of operation and patient exit from the OR were recorded contemporaneously by TNs using the existing theatre management software (Galaxy

Theatre Management System, iSOFT, DXC Technology, Virginia, US). Accuracy of timings was ensured by a trained observer (IJMM). Timings of patient entry and exit from the LS were recorded by the ophthalmologist performing the laser treatment. Start of operation and end of operation were defined as application of antiseptic solution to the eye and skin, and removal of eyelid speculum. Due to the nature of the surgery, it was not possible to mask any of the surgical team to the treatment arm. 


\section{Economic Model}

The results from this were used as inputs for a hypothetical economic model, reported in a previous publication, to determine an estimation of the costs of cataract surgery ${ }^{11}$. This financial model has been described in greater detail in the previous publication but was based on averaged costs/values from 5 different NHS foundation trusts and 4 femtosecond laser manufacturers. This model was used to provide an estimation of the difference in cost per case of running a FLACS service as compared with a traditional cataract service. Furthermore if the results supported that a hub and spoke model could be run with more than two ORs, these iterations were also tested using the model.

\section{Statistics}

For the purposes of this study, the first two CPS and FLACS theatre lists each were excluded from analysis as they were scheduled with reduced patient numbers in order to allow theatre staff to

familiarize with the models. The final four theatre sessions of the study were run as mixed lists in order to facilitate the scheduling of the remaining research participants and to avoid under utilised theatre sessions. These final mixed lists were also excluded from analysis.

The primary outcome as per the study protocol were the relative costs of FLACS and CPS. However in light of inherent difficulties in accurate recordings of costs within a large tertiary ophthalmology service, it was determined that this would be replaced with the number of cases on FLACS and CPS lists and the duration of the operations. This current study study of 299 patients had a power of $99 \%$ to detect an effect size (d) of 0.5 for the numbers of participants included in this analysis with regards to duration of surgery with $\alpha=0.05$ and a two tailed analysis.

Baseline characteristics were summarised for each treatment arm. Results were analysed primarily as per intention to treat. Continuous data was reported using means and standard deviations if data appear Gaussian, or medians and inter-quartile ranges if not. Binary data was reported as frequencies and percentages and evaluated with Fischer's exact test. Student's t-tests were used for parametric data and the Mann Whitney U test for non-parametric. All statistical tests used a twosided $p$ value of $\alpha=0.05$ unless otherwise specified. Intra-operative complications were defined as any event that involved unintentional trauma to an ocular structure, requiring additional treatment, or havin a negative effect on participants' eyesight. 


\section{Results}

A total of 427 patients (427 eyes) were recruited for the study and randomized to receive FLACS or CPS. Twenty-seven were excluded or withdrew in advance of surgery. For the purpose of this study comparing FLACS in a hub and spoke model with dual CPS theatre lists 299 of 400 operations were included for analysis (Figure 2). Excluded patients included 57 patients who had had surgery on the first 2 of each theatre sessions for FLACS/CPS and 44 patients treated on mixed (CPS and FLACS) theatre lists. There were no significant differences between patients included and excluded for this analysis other than those excluded were on average 3 years older $(\mathrm{p}=0.01)$ and had

shallower anterior chambers by $0.17 \mathrm{~mm}(\mathrm{p}<0.01)$ (Table 2). Of the 299 eyes included in this analysis, 134 patients had received FLACS, and 165 patients underwent CPS. Baseline demographics for the FLACS and CPS groups are seen in Table 3. The only significant difference at baseline was the FLACS group had a longer axial length by $0.39 \mathrm{~mm}(\mathrm{p}=0.02) .5$ patients due to receive FLACS were treated with CPS due to the following reasons: palpebral aperture too narrow for patient interface $(n=3,2.2 \%)$, patient lack of compliance $(n=1,0.7 \%)$, administrative error $(n=1$, $0.7 \%)$.

\section{High volume theatre list participants}

\section{Patients not treated on designated high volume theatre lists}

\begin{tabular}{|c|c|c|c|}
\hline Male/female & $140 / 159$ & $41 / 60$ & 0.30 \\
\hline $1^{\text {st }}$ Eye/2 $2^{\text {nd }}$ Eye & $243 / 56$ & $87 / 14$ & 0.29 \\
\hline Right Eye/Left Eye & $164 / 135$ & $55 / 46$ & 1 \\
\hline Age (years) & $69.45 \pm 10.81$ & $72.42 \pm 8.6$ & 0.01 \\
\hline $\begin{array}{r}\text { Pre-operative best } \\
\text { corrected distance } \\
\text { visual acuity } \\
\text { (logMAR) }\end{array}$ & $0.60 \pm 0.51$ & $0.51 \pm 0.36$ & 0.10 \\
\hline $\begin{array}{r}\text { Spherical } \\
\text { equivalent } \\
\text { refractive error } \\
\text { diopters (D) }\end{array}$ & $-0.50 \pm 4.32$ & $-0.38 \pm 3.52$ & 0.80 \\
\hline Axial length (mm) & $23.78 \pm 1.39$ & $23.67 \pm 1.56$ & 0.51 \\
\hline $\begin{array}{r}\text { Anterior chamber } \\
\text { depth }(\mathrm{mm})\end{array}$ & $3.27 \pm 0.38$ & $3.10 \pm 0.55$ & $<0.01$ \\
\hline $\begin{array}{r}\text { Average predicted } \\
\text { PCR risk }\end{array}$ & $1.63 \% \pm 1.24$ & $1.63 \% \pm 0.71 \%$ & 1 \\
\hline
\end{tabular}

The 139 patients undergoing FLACS were treated during 8 hub-and-spoke sessions, involving 16 four hour theatre sessions. The 160 patients randomized to CPS were treated on 20 cataract theatre lists. The average OR utilisation of a hub-and-spoke session was $221 \mathrm{mins} \pm 21(92.05 \% \pm 8.71)$ with a median of 9 patients treated in each OR, while the average duration of a CPS list was $230 \pm 22$ min $(95.81 \% \pm 9.17)(\mathrm{p}<0.001)$ with a median of 8 patients treated per list. Patients receiving FLACS spent, a mean time of $5.85 \pm 1.99 \mathrm{~min}$ in the LS. $25 \%$ of FLACS theatre sessions over-ran the allotted 4 hours compared with $30 \%$ of CPS lists. Average theatre over-run was $5 \pm 2.16$ min for FLACS and 13.67 \pm 8.76 mins for CPS ( $\mathrm{p}=0.09)$. 
Staffing levels for both models can be seen in Table 4. The hub-and-spoke model required one additional OT to be present compared with the CPS. A comparison of the average times associated with each operation can be seen in Table 5 and Table 6.

\section{FLACS (BOTH ORS) CPS (BOTH ORS)}

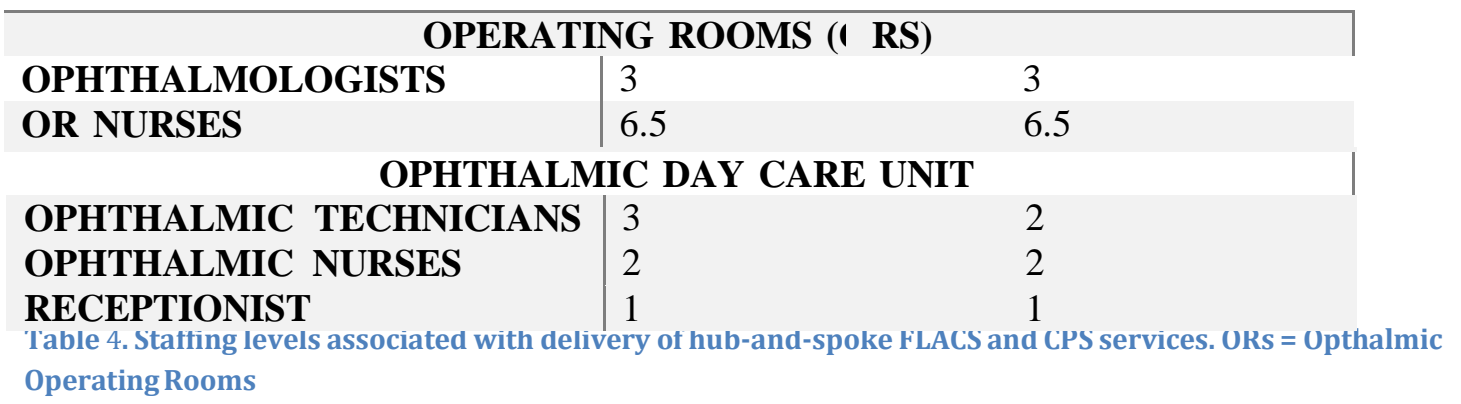

\begin{tabular}{|c|c|c|c|}
\hline & FLACS $(\mathrm{N}=139)$ & CPS $(\mathrm{N}=160)$ & $\begin{array}{l}\text { T TEST } \\
\text { (P VALUE) }\end{array}$ \\
\hline $\begin{array}{l}\text { TIME FROM ENTERING OR TO } \\
\text { START OF OPERATION }\end{array}$ & $5.83 \pm 2.58$ & $6.25 \pm 2.91$ & 0.19 \\
\hline DURATION OF OPERATION & $12.04 \pm 4.89$ & $14.54 \pm 6.19$ & $<0.001$ \\
\hline $\begin{array}{l}\text { TIME FROM END OF } \\
\text { OPERATION TO EXITING OR }\end{array}$ & $2.47 \pm 0.66$ & $2.6 \pm 1.02$ & 0.20 \\
\hline TOTAL TIME IN OR & $20.34 \pm 5.82$ & $23.39 \pm 6.89$ & $<0.001$ \\
\hline OR EMPTY & $5.27 \pm 3.25$ & $5.23 \pm 3.28$ & 0.92 \\
\hline
\end{tabular}

Table 5. Comparison of OR timings (in minutes) between hub-and-spoke FLACS and CPS based on intention to treat analysis. $\mathrm{OR}=$ Ophthalmic Operating Room

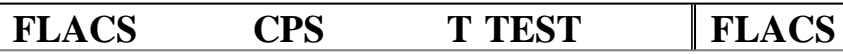




\begin{tabular}{|c|c|c|c|c|}
\hline & $(\mathrm{N}=134)$ & $(\mathrm{N}=165)$ & ( $\mathrm{p}$ value) & $\begin{array}{l}\text { CONVERTED TO } \\
\text { CPS }(\mathbf{N}=5)\end{array}$ \\
\hline $\begin{array}{l}\text { TIME FROM ENTERING } \\
\text { OR TO START OF } \\
\text { OPERATION }\end{array}$ & $5.82 \pm 2.62$ & $\begin{array}{l}6.24 \pm \\
2.87\end{array}$ & 0.19 & $6 \pm 1.58$ \\
\hline $\begin{array}{l}\text { DURATION OF } \\
\text { OPERATION }\end{array}$ & $11.73 \pm 3.53$ & $\begin{array}{l}14.71 \pm \\
6.76\end{array}$ & $<0.001$ & $20.40 \pm 17.87$ \\
\hline $\begin{array}{l}\text { TIME FROM END OF } \\
\text { OPERATION TO } \\
\text { EXITING OR }\end{array}$ & $2.47 \pm 0.66$ & $\begin{array}{l}2.59 \pm \\
1.02\end{array}$ & 0.24 & $2.4 \pm 0.89$ \\
\hline TOTAL TIME IN OR & $20.2 \pm 4.59$ & $\begin{array}{l}23.55 \pm \\
7.47\end{array}$ & $<0.001$ & $28.8 \pm 19.33$ \\
\hline OR EMPTY & $5.79 \pm 3.9$ & $\begin{array}{l}5.27 \pm \\
3.25 \\
\end{array}$ & 0.21 & $6.4 \pm 1.95$ \\
\hline
\end{tabular}

Table 6. Comparison of OR timings (in minutes) between hub-and-spoke FLACS and CPS based on actual operation peformed.

\section{Complications}

The overall rate of intraoperative complications were similar between the two groups $3.54 \%$ vs $3.76 \%$ however there was a noticeable difference in the rates of vitreous loss (0\% with FLACS compared to $1.88 \%$ in CPS) (Table 7).

\begin{tabular}{|l|ll|l|l|}
\hline \multicolumn{1}{|l}{ COMPLICATIONS } & FLACS & CPS & RR & P \\
\hline ANTERIOR CAPSULAR TEAR & $3(2.16 \%)$ & $3(1.88 \%)$ & $\begin{array}{l}1.15(95 \% \mathrm{Cl} \\
0.24-5.6)\end{array}$ & 0.86 \\
$\begin{array}{l}\text { POSTERIOR CAPSULE TEAR WITH } \\
\text { VITREOUS LOSS }\end{array}$ & $0(0 \%)$ & $3(1.88 \%)$ & $\begin{array}{l}0.16(95 \% \mathrm{Cl} \\
0.01-3.15) \\
3.45(95 \% \mathrm{Cl}\end{array}$ & 0.23 \\
$\begin{array}{l}\text { DM TEAR } \\
\text { SUPRACHOROIDAL HAEMORRHAGE }\end{array}$ & $1(0.72 \%)$ & $0(0 \%)$ & $\begin{array}{l}0.14-84.0) \\
3.45(95 \% \mathrm{Cl} \\
0.14-84.0)\end{array}$ & 0.45 \\
ABANDONED - EXTREME ZONULAR & $0(0 \%)$ & $1(0.63 \%)$ & $\begin{array}{l}0.38(95 \% \mathrm{Cl} \\
0.01-9.34)\end{array}$ & 0.56 \\
$\begin{array}{l}\text { WEAKNESS } \\
\text { TOTAL COMPLICATION RATE }\end{array}$ & $3.54 \%$ & $3.76 \%$ & $\begin{array}{l}0.95(95 \% \mathrm{Cl} \\
0.30-3.07)\end{array}$ & 0.94 \\
\hline
\end{tabular}

Table 7. Incidence of complications between the two treatment arms based on intention to treat analysis. *This patient was allocated to FLACS but was unable to undergo this procedure and the patient underwent CPS (RR = Relative Risk).

\section{Economic Modelling}

Based on our OR timings, using a hub-and-spoke model, we achieved a mean reduction of total time in the OR per patient of 3.05min. This allowed us to undertake one extra FLACS case per 4 hour theatre list compared to our CPS only lists. The average number of cases on using our operative models were were 8 for CPS and 9 for FLACS, which represented an average $12.5 \%$ increase in productivity. We applied these results to our economic model. Based on these results, the average cost for each cataract operation was $£ 355.42$ for CPS and $£ 500.02$ for FLACS (figure $3)$. 
A bivariate sensitivity analysis examining the number of cases/week and the cost of the patient interface (PI) was performed, reporting the additional cost per case of a FLACS service (Table 8).

\section{NUMBER OF CATARACT OPERATIONS PERFORMED PER WEEK}

\begin{tabular}{l|lllll} 
COST PER & & $\mathbf{2 0}$ & $\mathbf{4 0}$ & $\mathbf{6 0}$ & $\mathbf{8 0}$ \\
LASER & $\mathbf{4 0}$ & $£ 72.75$ & $£ 46.51$ & $£ 37.76$ & $£ 33.39$ \\
PATIENT & $\mathbf{7 0}$ & $£ 102.75$ & $£ 76.51$ & $£ 67.76$ & $£ 63.39$ \\
INTERFACE & $\mathbf{1 0 0}$ & $£ 132.75$ & $£ 106.51$ & $£ 97.76$ & $£ 93.39$ \\
$(£)$ & $\mathbf{1 3 0}$ & $£ 162.75$ & $£ 136.51$ & $£ 127.76$ & $£ 123.39$
\end{tabular}

Table 8. Bivariate sensitivity analysis comparing effects of cost of patient interface and number of operations performed per week on the additional cost of FLACS compared with CPS within a 2:1 hub-and-spoke FLACS model.

\section{3:1 and 4:1 Hub and spoke model}

Although the duration of FL application to the eye is usually between 25 and 45 seconds, patient time inside the laser room was 5.85 mins \pm 1.99 . In our model, the laser operator is an ophthalmologist working unassisted, and therefore the majority of time spent is on preparing the patient and setting up the laser. Based on our results, we recommend that the maximum number of ORs which could be run (in order to maximize the utility of a femtosecond laser) in a hub-andspoke model would be four (average total patient time in OR + turnaround time $25.12 \mathrm{mins} \pm 5.25$ ). Adding a third OR to our economic modelling of the costs of cataract surgery reduced the cost per case from $£ 500.02$ to $£ 477.28$ and adding a fourth reduced it to $£ 465.91$. Performing the same bivariate analyses on a 3:1 and 4:1 hub-and-spoke models as above shows that the difference in cost could be reduced further if the hospital was performing greater numbers of cataract surgery and received a significant discount in the cost of the patient interface from the manufacturer (Table 9). However in order to break even, our financial modelling shows the manufacturers would need to offer between 78-99\% discount on the cost of the PIs (Table 10Error! Reference source not found.).

Number of operations performed per week

\begin{tabular}{c|rr|rrrr}
\hline & & & 40 & $\mathbf{6 0}$ & $\mathbf{8 0}$ & $\mathbf{1 0 0}$ \\
\cline { 3 - 5 } $\begin{array}{c}\text { Cost per } \\
\text { patient }\end{array}$ & $\mathbf{3 : 1}$ & $\mathbf{4 0}$ & $£ 33.40$ & $£ 24.65$ & $£ 20.28$ & $£ 17.65$ \\
interface & Model & $\mathbf{7 0}$ & $£ 63.40$ & $£ 54.65$ & $£ 50.28$ & $£ 47.65$ \\
& & $\mathbf{1 0 0}$ & $£ 93.40$ & $£ 84.65$ & $£ 80.28$ & $£ 77.65$ \\
& & $\mathbf{1 3 0}$ & $£ 123.40$ & $£ 114.65$ & $£ 110.28$ & $£ 107.65$ \\
& & & Number of operations performed per week \\
& & & $\mathbf{4 0}$ & $\mathbf{6 0}$ & $\mathbf{8 0}$ & $\mathbf{1 0 0}$ \\
& $\mathbf{4 : 1}$ & $\mathbf{4 0}$ & $£ 26.84$ & $£ 18.10$ & $£ 13.72$ & $£ 11.10$ \\
& model & $\mathbf{7 0}$ & $£ 56.84$ & $£ 48.10$ & $£ 43.72$ & $£ 41.10$ \\
& & $\mathbf{1 0 0}$ & $£ 86.84$ & $£ 78.10$ & $£ 73.72$ & $£ 71.10$ \\
& & $\mathbf{1 3 0}$ & $£ 116.84$ & $£ 108.10$ & $£ 103.72$ & $£ 101.10$
\end{tabular}

Table 9, Bivariate sensitivity analyses demonstrating the additional cost of FLACS compared with CPS within a theoretical $3: 1$ and $4: 1$ hub-and-spoke FLACS model when the cost of the patient interface and the number of operations per week are varied. 


\begin{tabular}{r|lrrrrr} 
\% DISCOUNT & $\mathbf{2 0 0 0}$ & $\mathbf{3 0 0 0}$ & $\mathbf{4 0 0 0}$ & $\mathbf{5 0 0 0}$ & $\mathbf{6 0 0 0}$ \\
ON COST OF PI & $\begin{array}{l}\text { 4:1 } \\
\text { model } \\
\mathbf{3 : 1}\end{array}$ & 91 & 84 & 81 & 79 & 78 \\
$\begin{array}{l}\text { model } \\
\mathbf{2}: \mathbf{1} \\
\text { model }\end{array}$ & 96 & 89 & 86 & 84 & 82 \\
& n/a & 99 & 95 & 93 & 92 \\
Table 10. Break even points for hub-and-spoke FLACS with CPS services calculated for the \% discount of the patient
\end{tabular}

interface based on the number of ORs concurrently run and the number of operations performed per year

\section{Cases unable to undergo FLACS}

Five $(3.6 \%)$ patients randomised to FLACS did not receive femtosecond laser treatment. This is consistent with reported rates of unsuccessful attempts at FLACS between 2.3-6.3\% ${ }^{16-18}$. In our experience, the most common reason was that the palpebral aperture was too narrow to permit the $16 \mathrm{~mm}$ patient interface to applanate with the cornea. Four of these patients underwent uneventful CPS, with one suffering a suprachoroidal haemorrhage. This patient was considered at increased risk for this rare complication with an axial length of $19.66 \mathrm{~mm}$. 


\section{Discussion}

We have conducted a large randomised controlled trial comparing FLACS with CPS, by reporting the clinical outcomes and testing the efficiency of FL-centric methods of running cataract theatre lists. When FLACS is performed within traditional models featuring one surgeon, or installing the FL in the OR, productivity may be adversely affected, leading to incurring additional indirect costs ${ }^{8-}$

${ }^{10}$. This is because the duration of the patient's experience is increased with FLACS compared with CPS (time in OR + LS = 26.05 for FLACS vs 23.55 for CPS) Evaluating new models of delivering cataract surgery (such as a hub-and-spoke model) within a RCT, where patients are prospectively randomised to CPS or FLACS, allows us to test the model within a rigorous framework, rather than performing a case-control study where bias may be inherent.

By deploying a FL in the anaesthetic room adjacent to the OR and using a hub-and-spoke model, we have found that surgical time and patient time in OR are shorter for FLACS than CPS. Transfering some of the surgical steps into the LS reduces patient time in OR by 3.05minutes for FLACS ( $\mathrm{p}<0.001)$ led to an average of one extra operation per OR operating list of FLACS (median 9 cases per list) than CPS (median 8 cases per list), resulting in a $12.5 \%$ improvement in productivity (overall 2 more operations per session). Furthermore, despite the additional cases, the FLACS lists were shorter than the CPS lists, and more CPS sessions overran. Our method of titrating the numbers of patients scheduled for surgery to maximise the number of operations within the four hour session resulted in extremely high levels of OR utilisation. Our ambition resulted in a number of theatre sessions overrunning (25\% FLACS vs 30\% CPS), especially when unforeseen complications had occurred. Theatre list overruns may incur financial penalty at some NHS/public hospitals, however, our greater concern was to test the limits of our models.

There were some differences between the 299 patients included for this analysis and the 101 excluded, namely that the excluded group were 3 years older on average, with a corresponding shallower ACD by $0.17 \mathrm{~mm}$. It is unlikely that these differences are clinically significant and would have had a material effect on the timings of the theatre list ${ }^{15}$. Importantly, the prospectively calculated risk of PCR were equivalent between the two groups.

The mean time of each patient undergoing patient preparation for FL and FL application was 5.85 \pm 1.99 mins. Based such results it is easily possible to have a hub-and-spolke model of one FL feeding into 3 or 4 ORs (4:1 or 3:1) rather than 2 (2:1). we recommend that the ideal number of ORs to maximize the utility of a femtosecond laser in a hub-and-spoke model would be four (average total time per patient in OR + turnaround 25.12mins \pm 5.25 ). We predict that this would result in 3 or 4 more operations performed overall by the FLACS model per session (one for each OR).

Potential issues of having a 3:1 or 4:1 model to attempt to use a femtosecond laser as a tool for high volume surgery is that this requires a suitable and dedicated room within theatres and multiple ORs. This limits the use of such a model to an institution with such facilities already in place or a purpose-built unit (thus incurring additional costs). For instance, with a maximum of 2 eye ORs at our institution we are not able to evaluate adding additional ORs to our existing hub-and-spoke model. It is important to incorporate any development costs in the planning process if deciding whether to adopt this technology.

In order to minimize costs of running the hub and spoke model it is important to minimise the additional number of staff needed. Our model required 2 additional members of staff, one ophthalmologist to operate the laser and one OT to chaperone the patients between the LS and ORs. For our CPS lists, the extra OT was not present, however there were 3 ophthalmologists between 
the two ORs. This may have improved the efficiency of the CPS lists in our study to a degree by allowing the surgeons to rotate. The cost of the $3^{\text {rd }}$ surgeon was included in the financial modelling for the CPS model so as not to bias the model further in favour of CPS. In the FLACS model, it was also possible to permit surgeon rotation between the ophthalmologists performing laser and operating, thus possibly reducing the risk of surgeon fatigue during high volume cataract surgery.

In a previous publication we performed hypothetical financial modelling of hub-and-spoke delivered FLACS. Using a 2:1 model we found using bivariate sensitivity analyses that (for example) a $43 \%$ improvement in productivity would need to be achieved and accompanied by a $52 \%$ discount on the PI for the service to break even. This improvement in productivity was not realised by our study with a time saving of 3 minutes per patient (in the OR). The financial model demonstrates that the PI is the single most expensive item for the FLACS service. However, thus far FL platforms have tended to be used, and marketed as a premium product (based on reported improved refractive outcomes and stability) ${ }^{19}$. However it is very likely that a public health care service may be able to negotiate discounts on the costs of FLACS, especially if used within a highvolume service (which futher improves affordability) (Table 8 and Table 9). Further cost savings may be made by improved safety which may make cost savings in post-operative management ${ }^{20}$. It is important to note therefore that whilst there was no difference in posterior capsular rupture (PCR) and vitreous loss rates in this arm of the present study to investigate comparative high volume huband-spoke FLACS and CPS theatres lists (table 7), the results of the overall RCT showed a statistically significant reduction in PCR with FLACS, in a public health setting with different grade of surgeons operating, including those in training (personal communication $\mathrm{H}$. Roberts, D. O'Brart). As such complications incur additional costs, if our findings with respect to PCR rates are replicated by others, then our economic modelling might be more favourably inclined torward a FLACS huband-spoke model.

Our investigation of the effects of number of allied health professionals (AHPs) assisting in cataract theatre lists on the overall productivity showed a marked difference in the number of cataract surgeries performed between different institutions, but furthermore that a minimum of 4 AHPs are required to deliver high volume cataract surgery with effective use of theatre time and minimum delays $^{21}$. Our cataract ORs are generally run with 3 AHPs, which precludes further increases in productivity, which is evident in our turnaround time compared with other surgical units. The average time between one operation finishing and the start of the next was $13.57 \mathrm{~min}$ and $14.08 \mathrm{~min}$ for FLACS and CPS respectively, meaning that only between $47.0-50.8 \%$ of OR time is spent engaged in surgery. Our previous time and motion studies showed that patient turnover with 4 AHPs present can be reduced to 9.70mins within NHS units. Including one additional AHP per OR

to our unit (at a cost of $£ 70$ per session) to facilitate patient turnover could provide a similar overall time saving as this femtosecond laser technology.

\section{Limitations}

We chose to consider the productivity difference in terms of number of operations per OR and found a $12.5 \%$ improvement in FLACS. However other methods of assessing productivity could have been chosen (for example number of cases per surgeon). However there are usually an abundance of cataract surgeons in a department compared with theatre time and space, making this more of a limiting factor. We did not incur any infrastructure costs in the installation of the laser into the anaesthetic room and so are unable to provide a representation of infrastructure costs into our model. We understand however that other surgical units have incurred significant costs during the installation of a femtosecond laser, so this is an important consideration. The surgical team was not masked to the treatment arms and this may be associated with performance bias. Another potential source of bias is that we had to book patients to theatre lists preemptively; having a busier list may have improved productivity. Nevertheless we aimed to combat this by a fair and transparent method of a run in period before the trial commenced to build experience with the 
model and titrating booking numbers depending on previous early finishes and overrunning. These potential biases may have been even more evident within a case control study, hence why an RCT methodology was preferred.

\section{Conclusion}

In summary, FLACS with a hub-and-spoke model was significantly faster than CPS, with patients spending less time in the OR. This enabled a slight improvement in productivity, but not sufficient to meaningfully offset the additional costs relating to FLACS. Further gains in productivity may have been achieved with a 3:1 or 4:1 hub-and-spoke model. 
Acknowlegements

The research was supported by the NIHR Diagnostic Evidence Co-operative (DEC) London. The views expressed are those of the author(s) and not necessarily those of the NHS, the NIHR or the Department of Health. 


\section{Legends for figures in the text}

Figure 1. St Thomas' Hospital's 'hub-and-spoke' model for FLACS

Figure 2. CONSORT flowchart of participant numbers through the study.

Figure 3. Comparison of weekly costs of FLACS vs CPS services. 


\section{References}

1. Nagy Z, Takacs A, Filkorn T, Sarayba M. Initial Clinical Evaluation of an Intraocular Femtosecond Laser in Cataract Surgery. J Refract Surg 2009;25:1053-1060. Available at: http://cdn2.hubspot.net/hub/60407/file-14879786-

pdf/docs/laser_cataract_surgery_article_journal_of_refractive_sx.pdf.

2. Ewe SYP, Abell RG, Oakley CL, et al. A Comparative Cohort Study of Visual Outcomes in Femtosecond Laser-Assisted versus Phacoemulsification Cataract Surgery. Ophthalmology 2015:1-5. Available at:

http://eutils.ncbi.nlm.nih.gov/entrez/eutils/elink.fcgi?dbfrom=pubmed\&id=26526634\&retmo $\mathrm{de}=\mathrm{ref} \& \mathrm{cmd}=$ prlinks.

3. Chee S-P, Yang Y, Ti S-E. Clinical outcomes in the first two years of femtosecond laserassisted cataract surgery. Am J Ophthalmol 2015;159:714-719.e2. Available at: http://eutils.ncbi.nlm.nih.gov/entrez/eutils/elink.fcgi?dbfrom=pubmed\&id=25634531\&retmo $\mathrm{de}=\mathrm{ref} \& \mathrm{cmd}=$ prlinks.

4. Abell RG, Kerr NM, Vote BJ. Femtosecond laser- assisted cataract surgery compared with conventional cataract surgery. Clin Exp Ophthalmol 2013;41:455-462.

5. Abell RG, Darian-Smith E, Kan JB, et al. Femtosecond laser-assisted cataract surgery versus standard phacoemulsification cataract surgery: outcomes and safety in more than 4000 cases at a single center. J Cataract Refract Surg 2015;2015:47-52. Available at: http://eutils.ncbi.nlm.nih.gov/entrez/eutils/elink.fcgi?dbfrom=pubmed\&id=25466483\&retmo de $=$ ref \&cmd=prlinks.

6. Roberts TV, Lawless M, Bali SJ, et al. Surgical outcomes and safety of femtosecond laser cataract surgery: a prospective study of 1500 consecutive cases. Ophthalmology 2013;120:227-233.

7. Day AC, Gore DM, Bunce C, Evans JR. Laser-assisted cataract surgery versus standard ultrasound phacoemulsification cataract surgery. Day AC, ed. Cochrane Database Syst Rev 2016;7:CD010735.

8. Abell RG, Vote BJ. Cost-effectiveness of femtosecond laser-assisted cataract surgery versus phacoemulsification cataract surgery. Ophthalmology 2014;121:10-16.

9. Bali SJ, Hodge C, Lawless M, et al. Early experience with the femtosecond laser for cataract surgery. Ophthalmology 2012;119:891-899.

10. Lubahn JG, Donaldson KE, Culbertson WW, Yoo SH. Operating times of experienced cataract surgeons beginning femtosecond laser-assisted cataract surgery. J Cataract Refract Surg 2014;40:1773-1776. Available at:

http://eutils.ncbi.nlm.nih.gov/entrez/eutils/elink.fcgi?dbfrom=pubmed\&id=25217069\&retmo $\mathrm{de}=\mathrm{ref} \& \mathrm{cmd}=$ prlinks.

11. Roberts $\mathrm{H}, \mathrm{Ni} \mathrm{MZ}, \mathrm{O}$ 'Brart DPS. Financial modelling of femtosecond laser-assisted cataract surgery within the National Health Service using a "hub and spoke" model for the delivery of high-volume cataract surgery. BMJ Open 2017;7:e013616-9.

12. Wang L, Zhang Z, Koch DD, et al. Anterior chamber interleukin $1 \beta$, interleukin 6 and prostaglandin E2 in patients undergoing femtosecond laser-assisted cataract surgery. $\mathrm{Br} \mathrm{J}$ Ophthalmol 2016;100:579-582. 
13. Schultz T, Joachim SC, Kuehn M, Dick HB. Changes in prostaglandin levels in patients undergoing femtosecond laser-assisted cataract surgery. J Refract Surg 2013;29:742747.

14. Organisation TWH. WHO surgical safety checklist. 2009:1-1. Available at: http://www.who.int/patientsafety/safesurgery/checklist/en/.

15. Narendran N, Jaycock P, Johnston RL, et al. The Cataract National Dataset electronic multicentre audit of 55567 operations: risk stratification for posterior capsule rupture and vitreous loss. Eye 2008;23:31-37.

16. Day AC, Dhallu SK, Maurino V, Wilkins MR. Initial experience using a femtosecond laser cataract surgery system at a UK National Health Service cataract surgery day care centre. BMJ Open 2016;6:e012078.

17. Brunin G, Khan K, Biggerstaff KS, et al. Outcomes of femtosecond laser-assisted cataract surgery performed by surgeons-in-training. Graefes Arch Clin Exp Ophthalmol 2017;255:1-5.

18. Chang JSM, Chen IN, Chan W-M, et al. Initial evaluation of a femtosecond laser system in cataract surgery. J Cataract Refract Surg 2014;40:29-36. Available at: http://linkinghub.elsevier.com/retrieve/pii/S0886335013013047.

19. Conrad-Hengerer I, Sheikh AI M, Hengerer FH, et al. Comparison of visual recovery and refractive stability between femtosecond laser-assisted cataract surgery and standard phacoemulsification: Six-month follow-up. J Cataract Refract Surg 2015;41:1356-1364.

20. Qatarneh D, Mathew RG, Palmer S, et al. The economic cost of posterior capsule tear at cataract surgery. $\mathrm{Br} \mathrm{J}$ Ophthalmol 2012;96:114-117.

21. Roberts $\mathrm{H}$, Myerscough J, Borsci S, et al. Time and motion studies of National Health Service cataract theatre lists to determine strategies to improve efficiency. $\mathrm{Br} \mathrm{J}$ Ophthalmol 2017: bjophthalmol-2017-310452-10. 


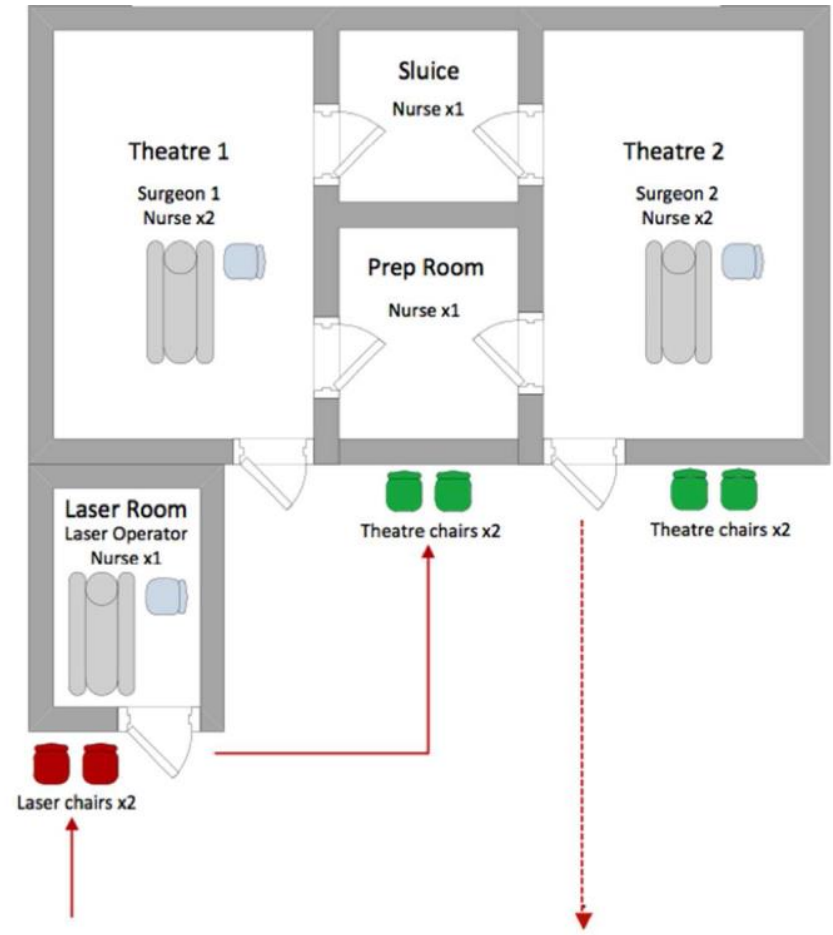

Figure 1. St Thomas' Hospital's 'hub-and-spoke' model for FLACS

$352 \times 264 \mathrm{~mm}(72 \times 72 \mathrm{DPI})$ 


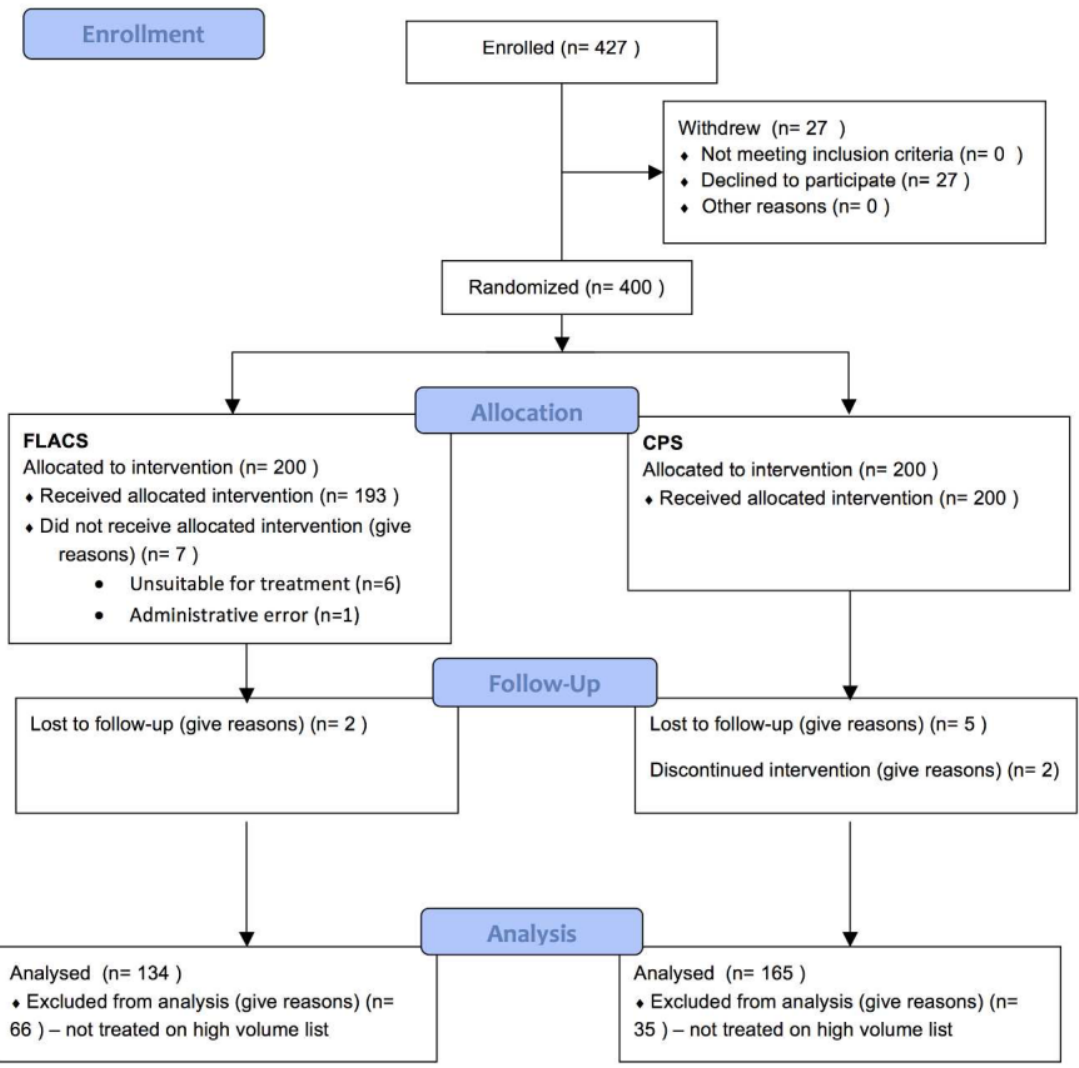

$215 \times 279 m m(300 \times 300$ DPI $)$ 


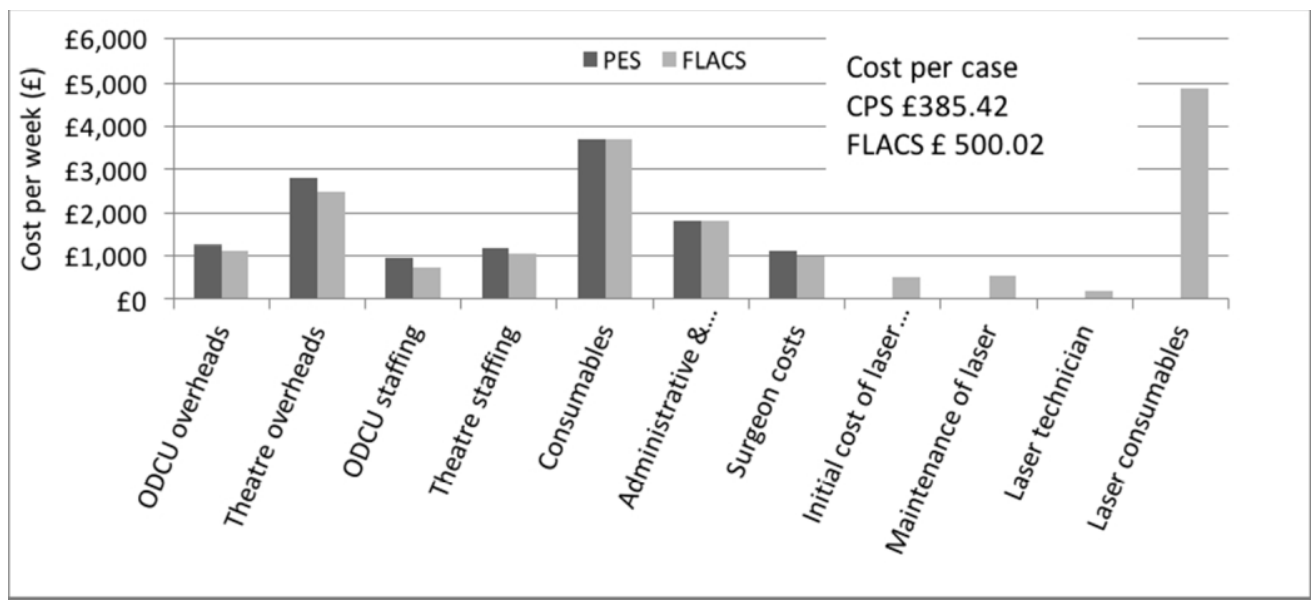

$159 \times 72 \mathrm{~mm}(150 \times 150 \mathrm{DPI})$ 